\section{A Novel High-Speed Optical Scanning Microscope for Routine Clinical} Applications

\section{Frekers ${ }^{1,2}$, I. Aksit1,2, V. Pilipenko'1, K. Bünger² ${ }^{1}$ Westfälische Wilhelms-Universität Münster, Germany, ${ }_{2}^{2} \mathrm{MedXP-GmbH}$, Gelsenkirchen, Germany Frekers@uni-muenster.de}

New and novel fast scanning microscopes with much enhanced image capturing and image analysis tools can dramatically extend the analytical power of traditional light microscopy by converting it to what one may call "virtual microscopy" (VM). One of the most important application fields of VM is connected to medical pathology. Here one can introduce the concept even to routine diagnostics in the day-to-day clinical environment. Further, over an intranet network, VM allows quick and easy access to the "virtual slides" database at any time and at any place. Another important aspect is the prospect that long-term archiving in large regional hospitals will be greatly facilitated. VM, thus, lies in the general stream of modern developments towards a "Digital Clinic". However, image acquisition speeds for capturing full specimen slide at several 100x magnifications have been the major bottleneck towards this long-term goal.

This article presents a technical break-through for a HighThroughput-Scanning-Microscope in the field of traditional light microscopy. The system allows image acquisition and digitisation of large scanning areas, where single microscope image views are being acquired with a high acquisition speed and then combined into one overall image for further image analysis or "image-browsing." Image-browsing and image analysis is accomplished through an enhanced software package (ICAT-Browser), which can be further extended according to customer specifications. The key innovation is the enhancement of the scanning speed, which is realized through the Anti-Motion-Blurring-Image-Scanning system (AMBIS ${ }^{\circledR}$ ), which is depicted in Fig. 1. It allows continuous scanning without mechanical start-stop sequences of the object under view and without blurring of the acquired image. AMBIS ${ }^{\circ}$ can be applied to all common microscope technologies such as bright field, polarized light,

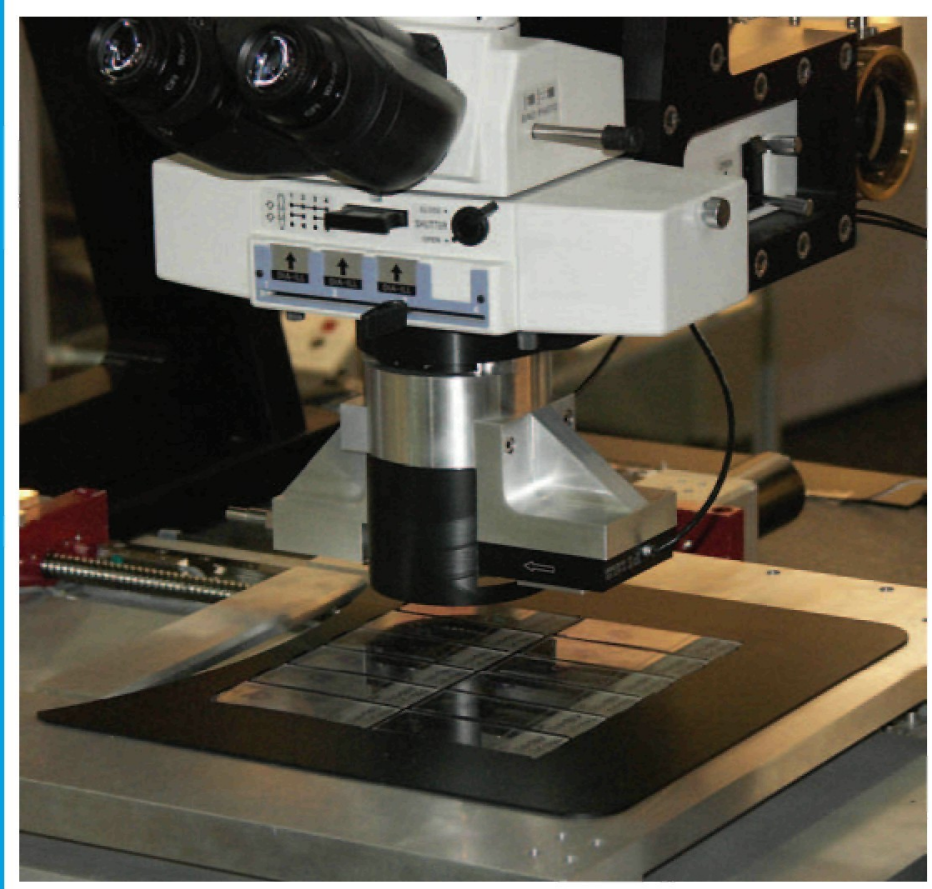

Figure 1. Anti-Motion-Blurring-Image-Scanning system (AMBIS $\left.{ }^{\oplus}\right)$. dark field, fluorescence, differential interference contrast, and phase contrast microscopy. In the present design, $\mathrm{AMBIS}^{\circ}$ features up to 50 frames per second (@3Mpx) in continuous scanning mode. The speed is limited by the bandwidth of the data bus and the intensity of the light source.

The intensity and homogeneity of the light source is, of course, a critical issue. We use Luxeon LED`s, which feature a light intensity of $200 \mathrm{~cd}$ with good emittance properties. Further, one can use these LED's in pulsed mode allowing an increase of the peak voltage beyond its nominal specification.

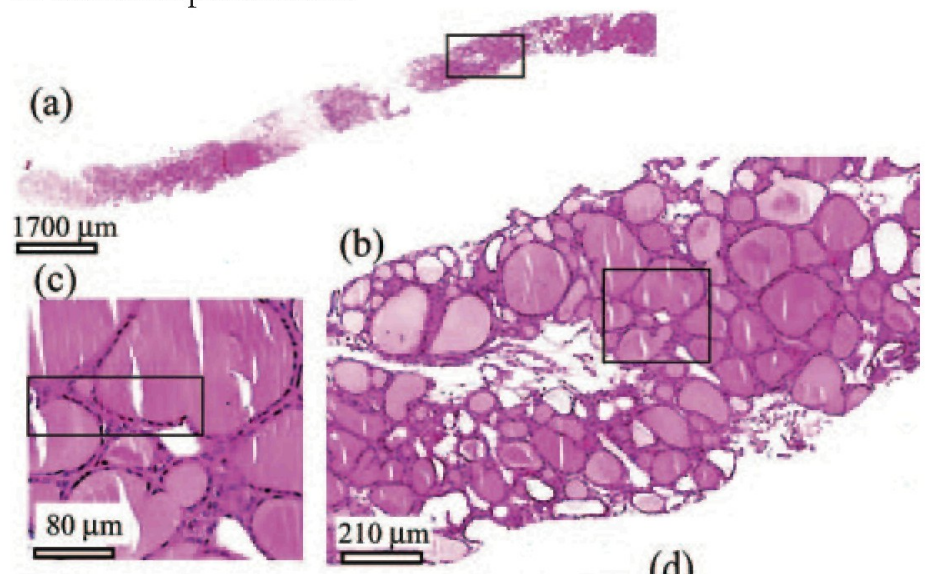

(d)

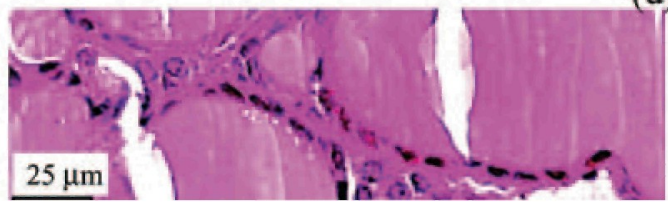

Figure 2. Digital microscopic images of the section of a thyroid with a 40x objective: (a) full image of sample; (b), (c) and (d) individual regions of interest as defined by ICAT cursor.

The presently developed microscope is equipped with a digital CMOS camera, a CAMERA-LINK ${ }^{\text {TM }}$ interface and a MATROX Odyssey frame grabber card with enhanced parallel compute power.

The implementation features a computer controlled $x / y$ crosstable with a stage position accuracy of $0.4 \mu \mathrm{m}$ over the full $195 \times 195$ $\mathrm{mm}^{2}$ table area. This area is large enough to scan about 20 slides in one loading operation. Additional techniques for feeding larger numbers of slides can readily be envisaged.

In the present scheme, the scanning process of a normal microscope slide can be accomplished in typically less than 120 seconds with $10 x$ objective, and in a few minutes with a $20 x$ objective. In addition, the system exhibits ultra-fast fine focusing with up to 100 focusing commands per second and a resolution of better than 0.1 $\mu \mathrm{m}$. There are additional software features like an image stitching function for seamless blending of overlapping neighboring images. Because of the continuous movement of the table, any adverse acceleration forces that would cause unwanted image offsets and discontinuities are avoided, making the rather computer-intensive stitching procedure almost obsolete. The lack of mechanical startstop sequences further minimizes the noise level, which one should regard as another positive side effect.

With the accompanying software it is possible to perform inspection and processing of regions of interest, or to navigate in $\mathrm{x} / \mathrm{y}$ direction over the scanned area. During scanning, the evolution of a global view can be observed in real time. Single and global images, groups of images or panorama overviews are stored in compressed mode (JPEG2000).

Figure 2 shows a digitised image of a thyroid sample with different magnified regions of interest, as they are defined by the cursor action. 


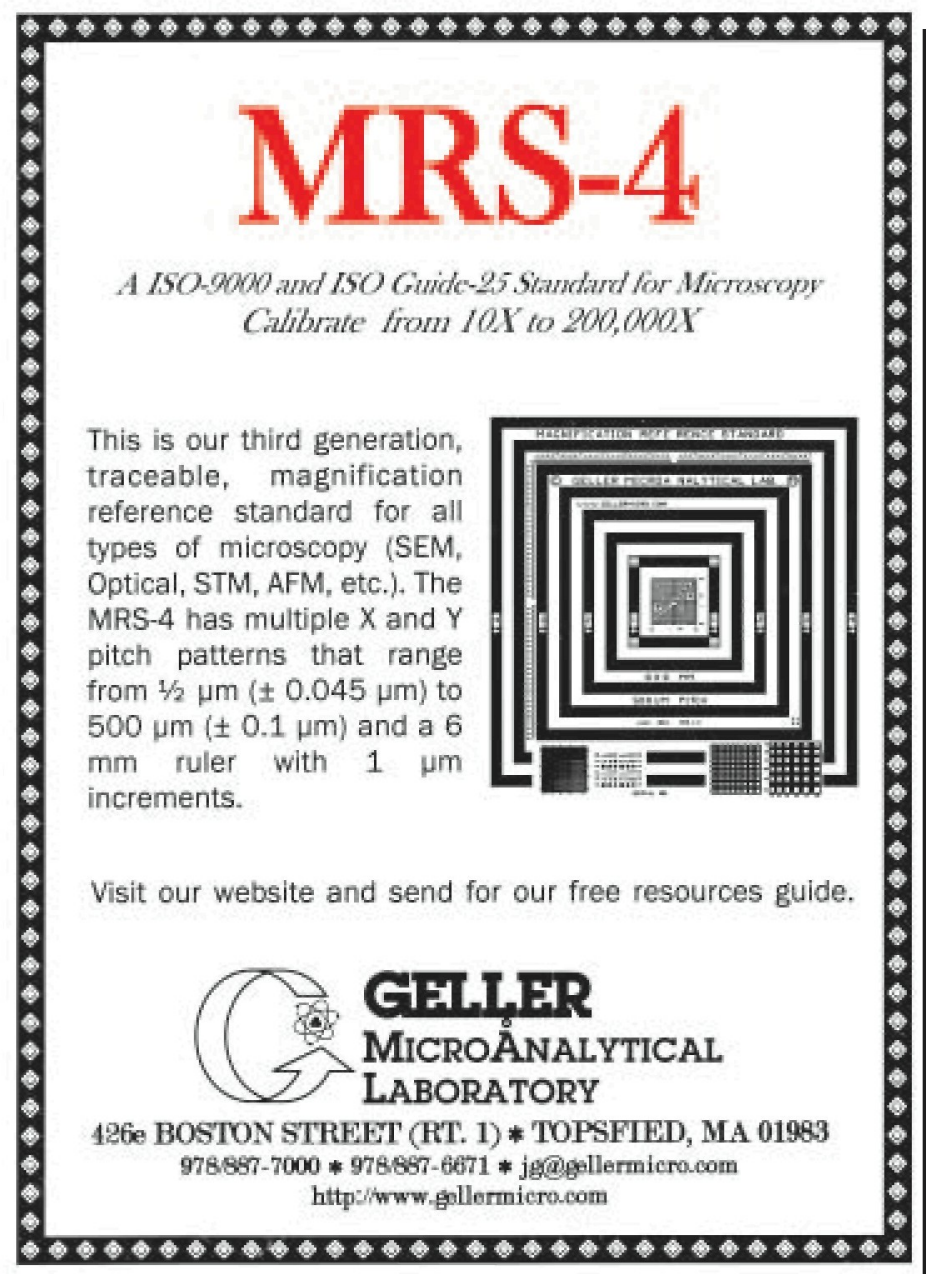

\section{TASTechnologies}

\section{A New Alternative to your TEM Specimen Holder service and repair needs.}

TGS Technologies provides High Quality TEM Specimen holder service with 25 years experience.

We offer competitive rates for our quality services.

\section{Contact Us Today}

TGS Technologies

Phone: 724.453 .3865

Fax: 724.453 .2968

E-mail: tom@tgstechnologies.net Web: www.tgstechnologies.net

\section{Quality Preparation Quality Results}

Advanced EM Specimen Preparation Instuments
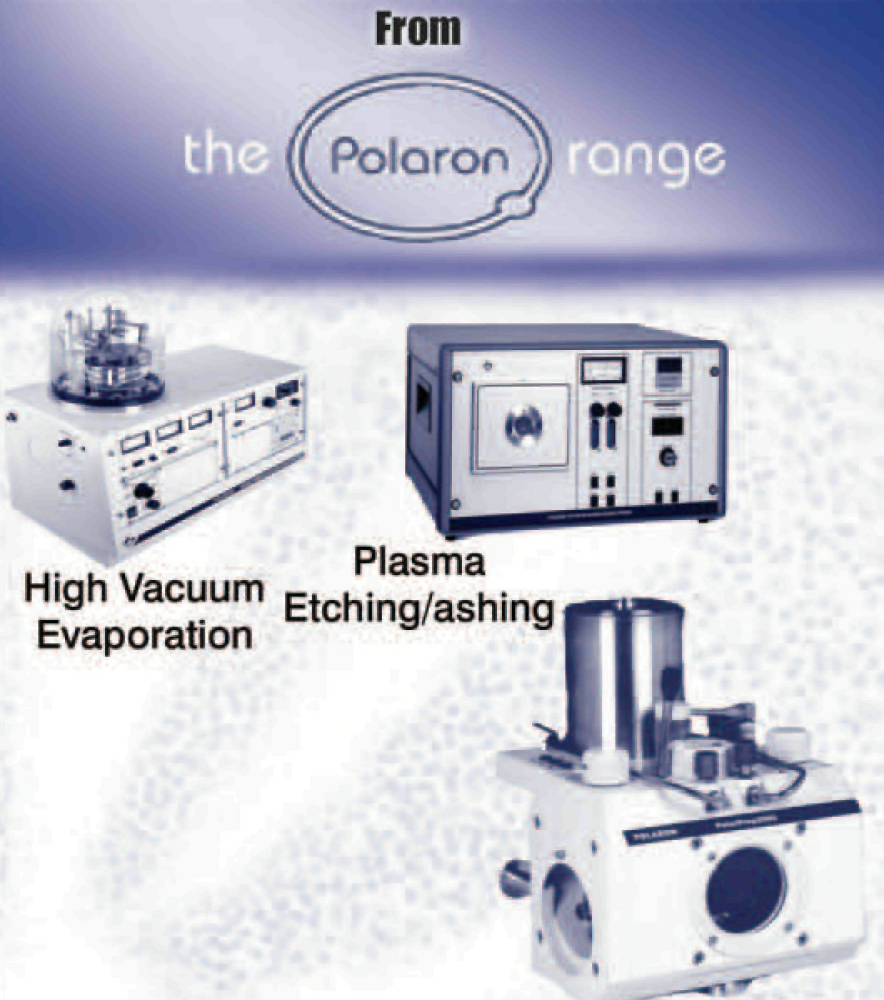

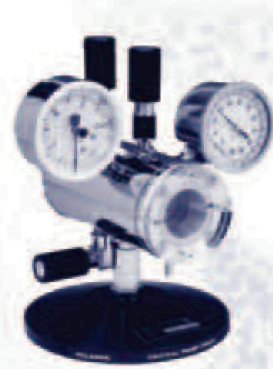

Critical Point Drying

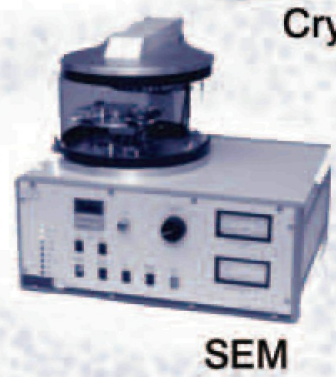

Coating

\section{Introducing the}

\section{E6500 Evanorator}

Low Gost - Full Features - Revolutionary Design

\section{$E B S_{\text {ciences }}$}

ADDING BRILIIANCE TO YOUR VISION

800-992-9037 or 413-786-9322

email: ebs@ebsciences.com www.ebsciences.com 